\title{
Optimizing a CVD-based Crystallization Process Using Neutral Particle Showering Deposition for Flexible Display
}

Received September 8, 2020; revised October 5, 2020; accepted October 12, 2020

\author{
Hyun-ki Kim and Byoung-deog Choi* \\ Department of Electrical and Computer Engineering, Sungkyunkwan University, Suwon 16419, Republic of Korea
}

*Corresponding author E-mail: bdchoi@skku.edu

\begin{abstract}
In this study, polycrystalline Si film was fabricated using a single-step Neutral Particle Showering Deposition (NPSD) process that can replace the composite process of low-temperature poly-Si. Accordingly, experiments were conducted on the effect of the gas treatment process on the crystallization of $\mathrm{Si}$ and film properties. After optimizing the gas atmosphere conditions in the process and the voltage applied to the reflector, we confirmed that the crystallization of the film was improved by temperature dependence deposition. During the deposition, $\mathrm{SiH}_{4}$ and $\mathrm{H}_{2}$ reaction gases were decomposed into the plasma; and activated or ionized $\mathrm{Si}$ and $\mathrm{H}$ supplied additional energy to the post-combination process when combined with the $\mathrm{SiO}_{2}$ structure on the sample surface. At this time, the energy of the neutral particle was the core of the collision energy of the particles. It was assumed that particle collisions were converted into thermal energy by vibration. We analyzed the effect of gas mixture ratio, metal reflector bias condition, and process temperature on the crystallization and properties of the Si thin film and found the optimized conditions for NPSD.
\end{abstract}

Keywords: Deposition temperature, Gas flow rate, Neutral particle, Neutral particle showering deposition, Raman, Reflector bias

\section{Introduction}

One of the core components of the display industry is the organic light emitting diode (OLED), which possesses several advantages, such as self-illumination. Among them, active matrix OLED (AMOLED) is currently used in displays of mobile phone and TV, due to its outstanding characteristics, such as a large range of light expression, no backlight requirement, excellent light quality, and fast response speeds compared to liquid crystal displays (LCDs). These displays also have a thin film transistor (TFT) backplane structure that drives each pixel [1]. Unlike the voltage-driven TFT-LCD, this method is current-driven. Therefore, these displays use poly-Si, i.e. low-temperature poly-Si (LTPS) and high-temperature poly-Si (HTPS), with a 100 times faster electron mobility than LCDs that use amorphous Si TFT in the backplane [2]. In the case of large-sized displays, oxide TFTs are employed. Concerning the realistic aspects such as production costs, AMOLED LTPS, which is known to have the best TFT characteristics and excellent image quality, are synthesized by converting the a-Si deposited via chemical vapor deposition (CVD) into partially crystallized poly-Si through low temperature $\left(\sim 600{ }^{\circ} \mathrm{C}\right)$ annealing using excimer laser annealing (ELA) [3]. However, this method has several drawbacks such as the non-uniformity of poly-Si, high cost of annealing equipment, and enlargement, which are yet to be overcome.

The deposition process using neutral particles and an additional energy source allow the growth of high-density films at low temperatures below $400{ }^{\circ} \mathrm{C}$ allowed by the glass substrates.

\section{Experimental details}

The process proposed in this study is capable of producing high-density thin films that cannot be obtained via conventional deposition processes and does not use additional heat sources under minimal temperature deposition conditions. In other words, it is a method of adding tertiary energy. In neutral particle showering deposition (NPSD) method, a cathode is connected to a reflector in a space where high-density plasma is formed under a high vacuum, cations in the plasma move to the reflector, collide, and become neutral particles. Since these neutral particles are not affected by the E and B fields, particles in the plasma and unscattered particles reach the sample and the kinetic energy of the particles is converted into thermal energy, which supplies the energy necessary for CVD deposition. Therefore, it is possible to form thin films even at room temperature and high-density thin films when the heat is applied to the substrate. Figure 1 shows the schematic of the NPSD process.

In this study, the process conditions needed to initiate crystallization using neutral particles were found and additional gas treatment was carried out to allow further crystallization. The preliminary conditions were modified by varying the gas ratio, process temperature, and reflector bias, to determine the degree of crystallization and optimize the process. All parameters in the process are listed in Table I. The power of the microwave generator was set to the maximum instrument power at $3 \mathrm{~kW}$. To measure of the crystallinity of the $\mathrm{Si}$ thin films, a Raman microscope with a $526 \mathrm{~nm}$ line laser is used as the 


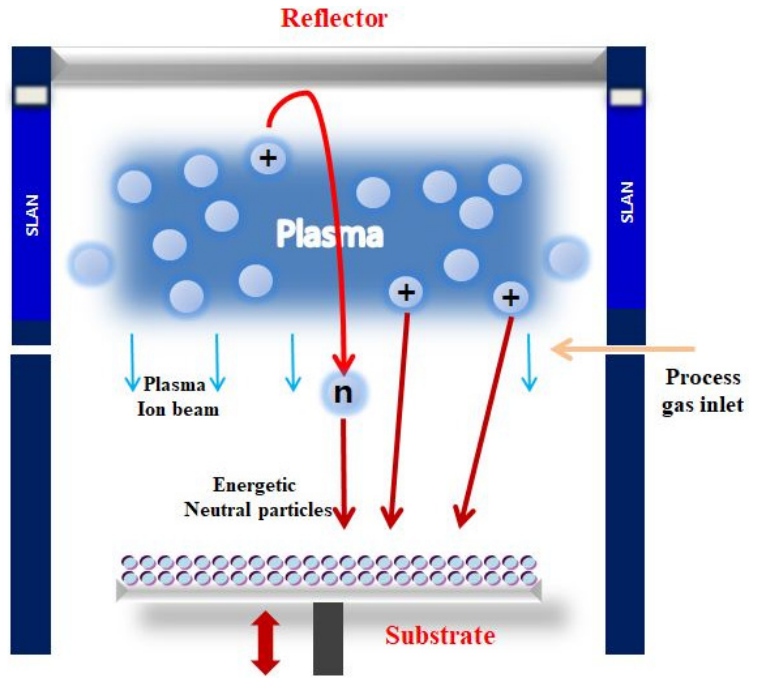

Figure 1. Schematic of the neutral particle showering deposition process.

Table I. Process variation recipe of NPSD.

\begin{tabular}{lcccc}
\hline & $\# 1$ & $\# 2$ & $\# 3$ & $\# 4$ \\
\hline $\mathrm{H}_{2}$ flow rate (sccm) & 0 & 20 & 40 & 60 \\
Reflector bias (V) & 0 & -10 & -20 & -30 \\
Process temp. ( $\left.{ }^{\circ} \mathrm{C}\right)$ & R.T (25) & 100 & 200 & $300 / 400$ \\
Process pressure & \multicolumn{5}{c}{$0.12 \mathrm{mTorr}$} \\
Microwave power & \multicolumn{5}{c}{$3 \mathrm{~kW}$} \\
\hline
\end{tabular}

excitation radiation. We used a laser power below $1 \mathrm{~mW}$ to prevent the formation of crystalline Si which occurs when using the high power of laser on the sample surface.

\section{Results and discussion}

\section{1. $\mathrm{SiH}_{4}: \mathrm{H}_{2}$ ratio condition}

The role of $\mathrm{H}_{2}$ in a c-Si deposition is pivotal. However, the large amount of $\mathrm{H}_{2}$ gas used for deposition releases independent $\mathrm{H}^{+}$ions that do not bond with the thin film; these $\mathrm{H}^{+}$ions act as hot carriers and degrade TFT properties. In a typical LTPS process, these $\mathrm{H}$ ions are removed through an additional dehydration process [4].

In the $\mathrm{Si}-\mathrm{Si}$ bonding process, the first step is $\mathrm{Si}-\mathrm{H}-\mathrm{Si}$ bonding and $\mathrm{Si}-\mathrm{Si}$ bonding is completed as the $\mathrm{H}$ ion escapes. $\mathrm{H}$ ion is indispensable in this process, and the higher the ratio of $\mathrm{H}_{2}$ gas, the easier the deposition of the $\mathrm{Si}$ film. However, as the $\mathrm{H}^{+}$ion concentration increases, the $\mathrm{H}$-occupancy in the film increase, which degrades the performance of the film. Therefore, to improve the performance of the film, $\mathrm{H}^{+}$ions should be reduced as much as possible within the limit of the deposition of the Si film. The $\mathrm{H}_{2}: \mathrm{SiH}_{4}\left(\mathrm{SiH}_{4}: \mathrm{He}=1: 9\right.$ mixed gas was used) ratio of 2:1 applied in this study is the minimum $\mathrm{H}_{2}$ ratio for $\mathrm{Si}$ film deposition and is a very small quantity [5-6]. In general, at a ratio of $\mathrm{H}_{2}: \mathrm{SiH}_{4}=4: 1$ or higher, the presence of $\mathrm{c}-\mathrm{Si}$ in the thin film was easily confirmed through a general plasma enhanced chemical vapor deposition (PECVD) process.

As can be seen from the Raman spectra in Fig. 2, optimal results were obtained with the $\mathrm{H}_{2}: \mathrm{SiH}_{4}$ ratio of 2:1. During the formation of

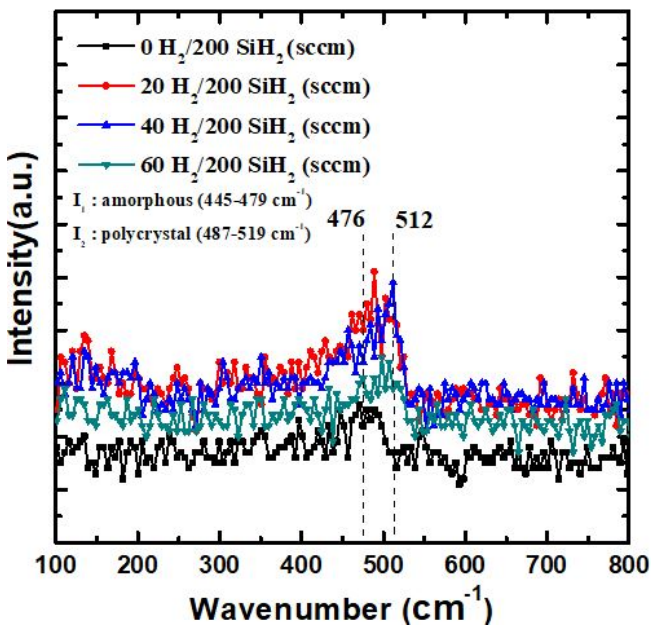

Figure 2. Raman results for various $\mathrm{H}_{2}$ : $\mathrm{SiH}_{4}$ ratios.

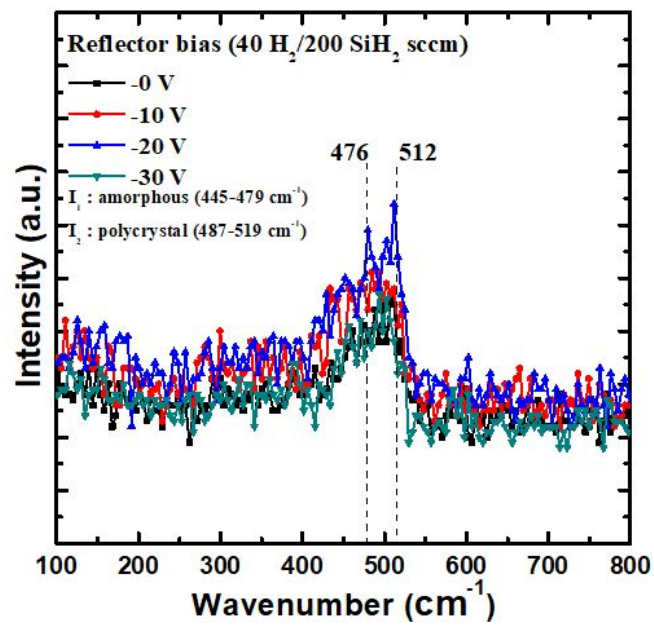

Figure 3. Raman results for various reflector bias values.

c-Si, we assumed that $\mathrm{H}_{2}$ gas was the dominant factor in the deposition reaction. In this case, it was known that the surplus $\mathrm{H}$ ions in the thin film and c-Si were limited to nanocrystalline structures or smaller. At a ratio of $\mathrm{H}_{2}: \mathrm{SiH}_{4}=3: 1\left(60 \mathrm{H}_{2} \mathrm{sccm}\right)$, it can be seen that the Raman peak value decreased and slight moved to the left.

\subsection{Metal reflector bias condition}

The bias of the metal reflector is important for determining the kinetic energy of the neutral particles. However, the applied bias should be adjusted within a range such that sputtering does not occur by ions like He.

The negative bias of the metal reflector formed a negative E-field and the cations present in the region near the metal reflector and the top of the plasma were preferentially pulled into the metal reflector under the influence of the negative E-field and collided. Figure 3 shows the Raman spectra for different reflector biases. Although the peak corresponding to c-Si $\left(520 \mathrm{~cm}^{-1}\right.$ peak) was dominant, a-Si peak (480 $\mathrm{cm}^{-1}$ peak) was also observed in some studies [7-9].

The above results imply that even if the deposition was performed using optimum neutral particle energy conditions, there 


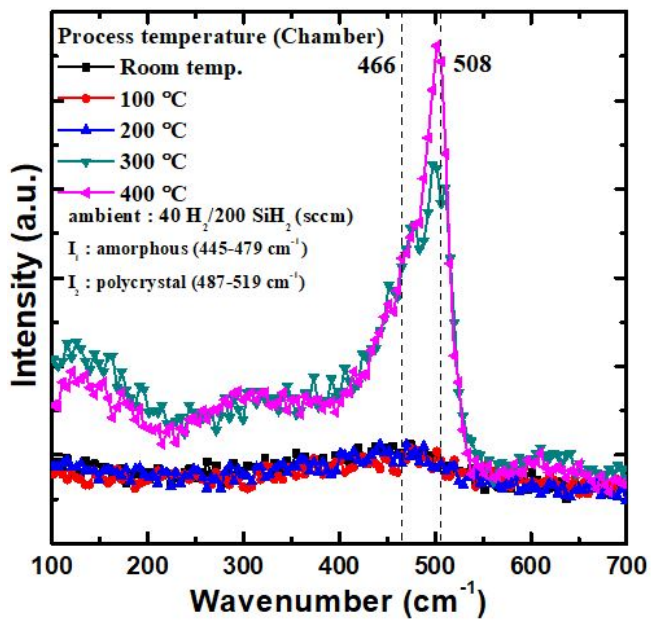

Figure 4. Raman results for various deposition temperatures.

may be a limit to complete the poly-Si deposition. It was inferred from Fig. 3 that neutral particles colliding with more than adequate kinetic energy affected the silicon crystallization process, where Si crystals degraded at $-30 \mathrm{~V}$. When the bias applied to the reflector exceeds a certain level, the kinetic energy of the neutral particles increases, breaking the Si-Si bonding where crystallization has progressed. This takes place as neutral particles, generated upon collision of cations in the plasma with the reflector, supply excessive energy to the film. To crystallize a sample by monitoring the particle energy high sample temperature is needed [10].

\subsection{Process temperature}

In Fig. 4, Raman spectra were recorded for depositions at process temperatures including room temperature (25), 100, 200, 300, and $400{ }^{\circ} \mathrm{C}$. The spectra were used for comparing the degree of $\mathrm{Si}$ crystallization within a thin film. Below $200{ }^{\circ} \mathrm{C}$, no Si crystals were formed. However, at $300{ }^{\circ} \mathrm{C}$ (Fig. 4), crystal formation started dramatically.

When the ion energy was $10 \mathrm{eV}$, the epitaxial layer yielded growth around $300{ }^{\circ} \mathrm{C}$, marking a distinct boundary between the crystal and amorphous phase [10-11]. The energy of He ions due to the $-20 \mathrm{~V}$ bias of the reflector could not be calculated accurately but considering that crystallization progressed above $300{ }^{\circ} \mathrm{C}$, it was comparable to the graph characteristics seen below $20 \mathrm{eV}$ and it is considered to be $15-20 \mathrm{eV}$. Also, since neutral particles do not carry a charge, they are not expected to be significantly affected by the sheath voltage drop. Therefore, the energy applied to the substrate is expected to be about $10 \mathrm{eV}$, which is reduced by half. The results from Raman spectroscopy revealed that temperature difference did have a significant effect on the thin film deposition process in the temperature range of 300 to $400{ }^{\circ} \mathrm{C}$.

\section{Conclusions}

By studying the CVD process, we confirmed the technical feasibility of a one-step NPSD process that could replace the LTPS process. We found the following optimum neutral particle annealing process conditions using an inert gas: the electron cyclotron resonance plasma condition that enables appropriate sample temperature and high vacuum plasma density. Typically, poly-Si deposition is not possible with conventional CVD methods at temperatures below $400{ }^{\circ} \mathrm{C}$. There have been many attempts to replace the LTPS process till now but most of them have only achieved results at the level of nanocrystalline $\mathrm{Si}$. In this study, the poly-Si deposition was observed below $400{ }^{\circ} \mathrm{C}$ by increasing the flux of plasma in the chamber. In addition, it is seen that poly-Si deposition is possible on any plastic substrate capable of $\mathrm{Si}$ deposition at $300{ }^{\circ} \mathrm{C}$. This study completed the first phase of the major challenge of replacing the high-cost composite process called AMOLED LTPS with a single process. Our study will motivate further attempts to completely replace the AMOLED LTPS with an efficient method.

\section{Acknowledgements}

This work was supported by Department of Electrical and Computer Engineering, Sungkyunkwan Univ., Suwon 16419, Republic of Korea and SSEMVIT Korea.

\section{References}

[1] C. W. Tang and S. A. VanSlyke, App. Phys. Lett. 51, 12 (1987).

[2] A. V. Shah, J. Meier, E. Vallat-Sauvain, N. Wyrsch, U. Kroll, C. Droz, and U. Graf, Sol. Energy Mater. Sol. Cells 78, 1 (2003).

[3] Y. Oana, J. Soc. Inf. Display 9, 3 (2012).

[4] C. G. Van de Walle and N.H. Nickel, Phys. Rev. B 51, 4 (1995).

[5] J. Robertson, J. Appl. Phys. 87, 5 (2000).

[6] J. N. Jang, B.C. Song, D. H. Lee, S. J. Yoo, B. Lee, and M. Hong, Thin Solid Films 519, 20 (2011).

[7] J. M. Owens, D. Han, B. Yan, J. Yang, K. Lord, and S. Guha, MRS Proc. 762, A4.5 (2003).

[8] Q. Cheng, S. Xu, and K. Ostrikov, J. Mater. Chem. 19, 29 (2009)

[9] D. Gracin, A. Gajović, K. Juraić, M. Čeh, Z. Remeš, A. Poruba, and M. Vaněček, J. Non-Cryst. Solids 354, 19 (2008).

[10] J. W. Rabalais, A. H. Al-Bayati, K. H. Boyd, D. Marton, J. Kulik, Z. Zhang, and W. K. Chu, Phys. Rev. B 53, 16 (1996).

[11] X. Zhou, M. Ishida, A. Imanishi, and Y. Nakato, J. Phys. Chem. B 105, 1 (2001). 